\title{
VIBRATIONAL RELAXATION DEMONSTRATED IN NICOTINAMIDE ADENINE DINUCLEOTIDE APPL YING MACHINE LEARNING BASED ANALYSIS
}

\author{
Áron Sipos ${ }^{1}$, Rita Nagypál ${ }^{1,2}$, Ferenc Sarlós ${ }^{1}$, Géza I. Groma ${ }^{1}$ \\ ${ }^{1}$ Institute of Biophysics, Biological Research Centre Szeged \\ ${ }^{2}$ Doctoral School of Physics, University of Szeged
}

DOI: https://doi.org/10.14232/kvantumelektronika.9.30

\section{Introduction}

Found in prokaryotic and eukaryotic cells, the metabolic cofactor nicotinamide adenine dinucleotide (NAD) plays an essential role in redox reactions, powering biochemical reactions as a major electron carrier [1-3]. Existing in its oxidized (NAD+) and reduced (NADH) forms, NAD is an important coenzyme and metabolic marker since any perturbation to the NADH/NAD+ ratio can be a good indicator of a diseased cell or disturbed cell cycle states $[1,4]$. While the oxidized form of NAD is nonfluorescent, it has been demonstrated that NADH exhibits autofluorescence showing a fluorescence band with a peak at $460 \mathrm{~nm}$ [5]. Composed of adenosine monophosphate, dihydronicotinamide ribose 5'- phosphate, two 5'-nucleotides and a pyrophosphate bridge, the structure of the reduced form NADH has been widely observed [6]. Although NMR and theoretical studies suggested several possible conformations, based on the work of Meyer et al. two main groups can be noted: an unfolded/extended form in which the nicotinamide group lies apart from the adenine and a folded form, the two bases are stacking upon each other [7-10]. The conformational heterogeneity of NADH can be described as a rapid, dynamic equilibrium of folded and unfolded states, the former one being described as dominant in aqueous solutions [7]. Based on time-resolved studies, upon direct excitation of its nicotinamide group multicomponent decay dynamics of NADH has been revealed, and upon exciting the chromophore group, fluorescence decay is fit by two exponentials, with 0.4 and $2.0 \mathrm{~ns}$ lifetimes, attributed to the free and the bound forms, respectively $[11,12]$. Regarding free $\mathrm{NADH}$, in aqueous solution biexponential fluorescence decay is exhibited showing the lifetimes of 0.3 and $0.7 \mathrm{~ns}[6,13,14]$. It is concluded that the lifetimes strongly depend on the solvent type and temperature [13-16]. Upon excitation at its adenine ring, studies suggested direct intramolecular energy transfer to the nicotinamide group with a high efficiency in aqueous solution and a diminished one in water-methanol solutions, attributed to denaturation $[6,8,10]$. In the present study we measure the fluorescence kinetics of NADH in an aqueous solution with high precision and apply a custom machine learning based analysis method to determine exactly the nature of the decay dynamics.

\section{Methods}

The fluorescence kinetics was measured by applying the combination of two different techniques (Figure 1). The $100 \mathrm{fs}-1 \mathrm{~ns}$ range was measured by fluorescence upconversion (UpConv), while the 100 ps - 10 ns range by time-correlated single photon counting (TCSPC). A laser oscillator (Mai Tai eHP, Spectra Physics) was used as the light source (central wavelength applied $750 \mathrm{~nm}$, mean power $2.5 \mathrm{~W}$, repetition rate $80 \mathrm{MHz}$, pulse length $\sim 70 \mathrm{fs}$ ), the dispersion of the setup was compensated by a prism pair. The laser beam was split to pump and gate beams using a half-wave plate and a polarization beam splitter. The pump beam was frequency doubled using a BBO crystal. The second harmonic ( $\mathrm{SH}$ ) was separated from the pump using two dichroic mirrors, and short pass color filters, and after focusing with lens $(f=+50 \mathrm{~mm})$ was used to excite the NADH. The polarization of the excitation was set using a half-wave plate. The fluorescence was collected after a wire grid polarization filter with a pair of off-axis parabolic mirrors (effective focal lengths 2" and 12"), and 
focused through a longpass color filter onto a sum frequency generating BBO crystal. The gate beam was driven through a delay line containing a retroreflector mounted to a precision translator (UTS150CC, Newport) and focused onto the sum frequency generating BBO with a $\mathrm{f}=+200 \mathrm{~mm}$ lens. The angle between the fluorescence and the gate beam was $11^{\circ}$. The generated sum frequency was driven into an imaging spectrometer (iHR550, JobinYvon), and was detected using a CCD camera cooled by liquid nitrogen (Symphony, JobinYvon). The time resolution of the UpConv setup was measured using the Raman signal of distillated water and was found to be 165 fs.

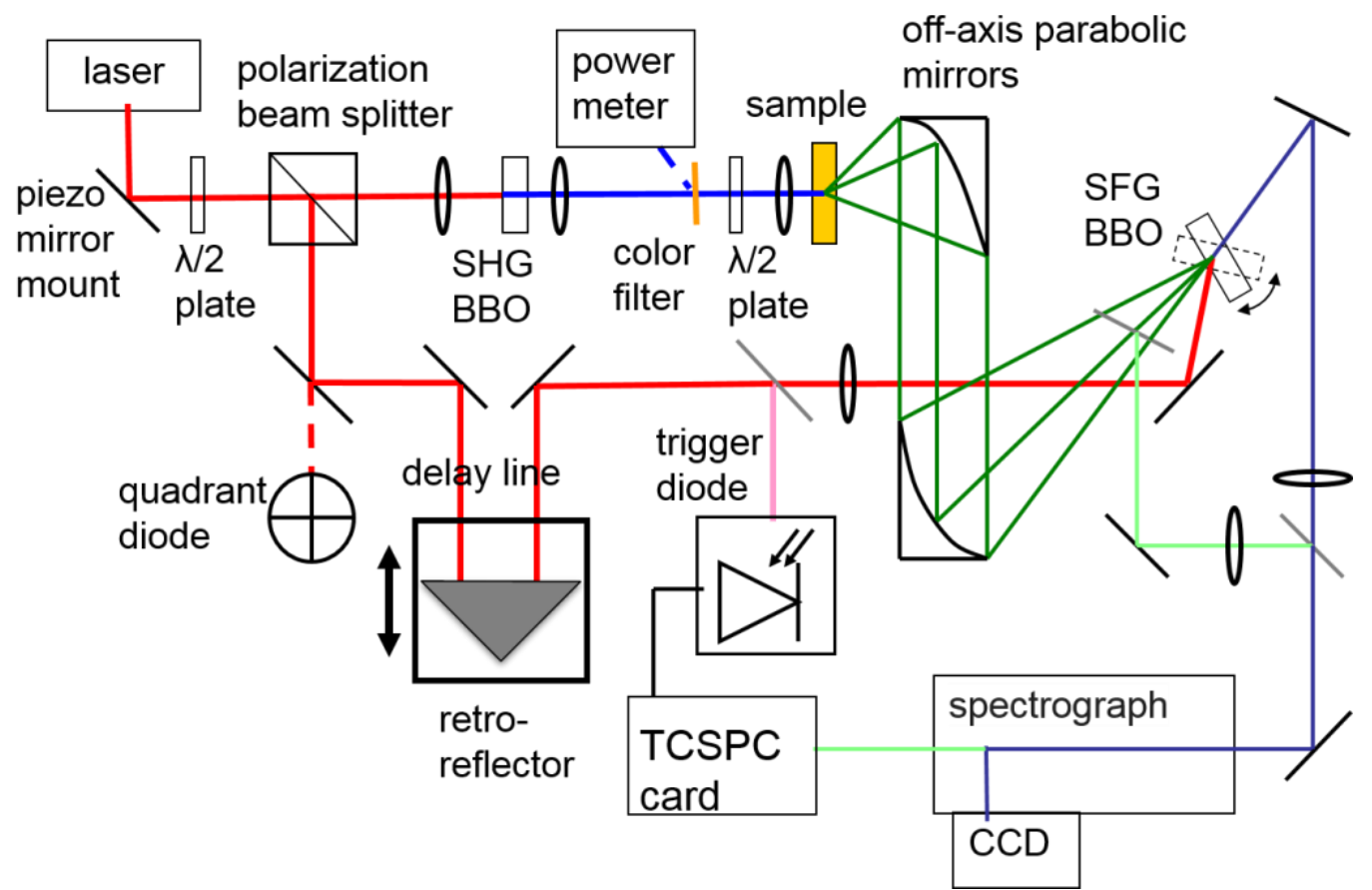

Figure 1. The layout of the experimental setup.

For the TCSPC measurement a Becker \& Hickl SPC-130 module was used. The fluorescence was weakened using ND filters, was driven directly into the spectrograph and the signal of the selected wavelength was detected using an avalanche diode (ID-100-50-UNL, ID Quantique). The gate beam was focused onto a fast photodiode (DET10M, Thorlabs) providing trigger signal for the TCSPC measurement. The time resolution of the TCSPC setup was 45-50 ps.

In the course of the measurements the direction of the laser beam and the SH power were stabilized. The beam direction was measured using a quadrant diode (SpotOn, Duma Optronics) and stabilized using a piezo mirror mount (KC1-PZ, Thorlabs). The SH power was measured at the reflection on the color filter using a photodiode power meter (PD300, Ophir), and was stabilized by the rotation of the half-wave plate at the polarization beam splitter.

A $3 \mathrm{mM}$ solution of NADH (Sigma-Aldrich, in the form of disodium salt hydrate) was prepared in $0.1 \mathrm{M}$ PIPES buffer $(\mathrm{pH}=7.0)$ and $15 \mathrm{mM}$ ascorbic acid was added to minimize the oxidation of that. The sample was circulated through a $1 \mathrm{~mm}$ quartz cuvette (Starna) by a peristaltic pump. The sample was cooled to $16^{\circ} \mathrm{C}$. The excitation power was $3 \mathrm{~mW}$ and the spot size at the sample was $\sim 50 \mu \mathrm{m}$. The cooling and the addition of ascorbic acid prevented NADH from oxidation in an extent that during the $\sim 15$ hour of UpConv measurements the signal did not decrease significantly, and this decrease was corrected for. The fluorescence kinetics were detected at magic angle at seven wavelengths in the 450-570 nm wavelength range.

The kinetics obtained by the combination of the data detected in the UpConv and TCSPC measurements are presented in Figure 2. 


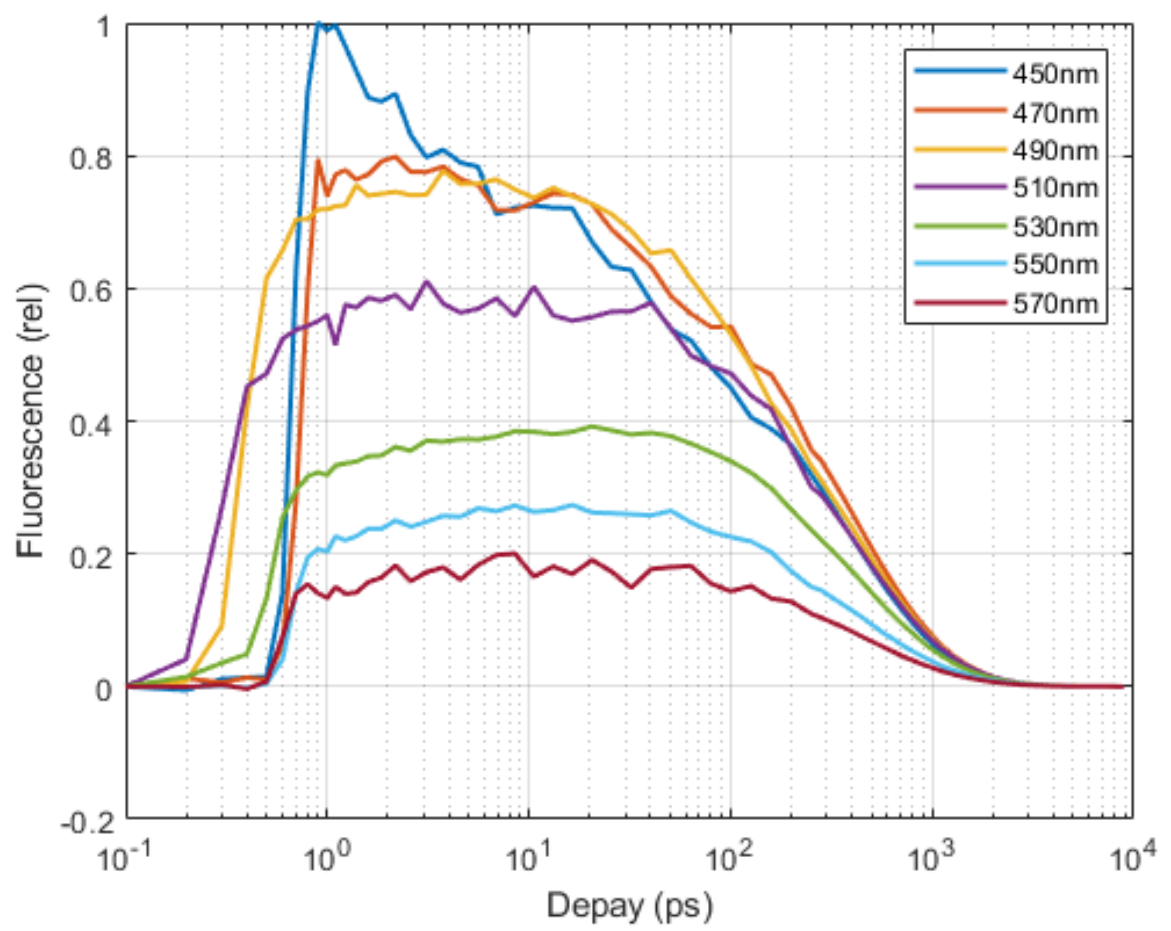

Figure 2. The fluorescence kinetics of NADH at different wavelengths.

\section{Analysis of data}

Kinetic datasets are challenging to analyze due that many reactions could take place simultaneously, and it is hard to distinguish them. A reason for this complexity is originated in the heterogeneity of the conformational states of a fluorescent molecule studied by time-resolved fluorescence $[17,18]$. One could assume that the data could be approximated by first-order kinetics, but in many cases we do not have proper knowledge on whether this assumption is true or not. In our case, the data do not hold direct information on the time-dependent concentration of the different compounds created by the photoexcitation, what we have is a set of kinetics measured at different wavelengths. The straightforward method in this case is to fit the kinetics by a sum of exponential terms. Unfortunately this type of analysis is also problematic, for eg. the number of components is not known in advance and the experimental data may be taken relatively sparsely over a long period of time. It was shown earlier [19, 20], that a superior way for the analysis is the application of the LASSO (Least Absolute Selection and Shrinkage Operator) method defined as

$$
\operatorname{minimize}\left[\frac{1}{2}\|\mathbf{b}-\mathbf{A} \mathbf{x}\|_{2}^{2}+\lambda\|\mathbf{x}\|_{1}\right] .
$$

Here $\mathbf{b}$ is the vector of the experimental data with length of $m$, the element $b_{i}$ of which is taken at the time $t_{i}, \mathbf{A}$ is an $m \times n$ matrix - called the design matrix - with elements of

$$
A_{i j}=\exp \left(-t_{i} / \tau_{j}\right),
$$

where $\tau_{j}$ is an element of the vector $\tau$ of length $n$, consisting of a series of pre-defined time constants, $\mathbf{x}(\boldsymbol{\tau})$ is the distribution to be determined and $\lambda$ is a positive hyperparameter. This method ensures that solution $\mathbf{x}(\boldsymbol{\tau})$ will be sparse. Furthermore, because we can state that at the different wavelengths of the observations we have the same conformations and states of the NADH molecule - although it is clearly observable that the decays are different - we can suppose a strong correlation among them. This type of correlation can be achieved by an extension of the LASSO, called the group LASSO. Another extension, the elastic net, containing a second ( $L_{2}$ type) penalty term with a $\omega$ 
hyperparameter makes possible to control the width of the peaks in the solution. Utilizing the statistical methods of cross validation (CV) and Bayesian optimization (BO), we set up a machine learning algorithm to select the solutions automatically. The task of the algorithm is to find the $(\lambda, \omega)$ parameter pair based on the experimental data only.

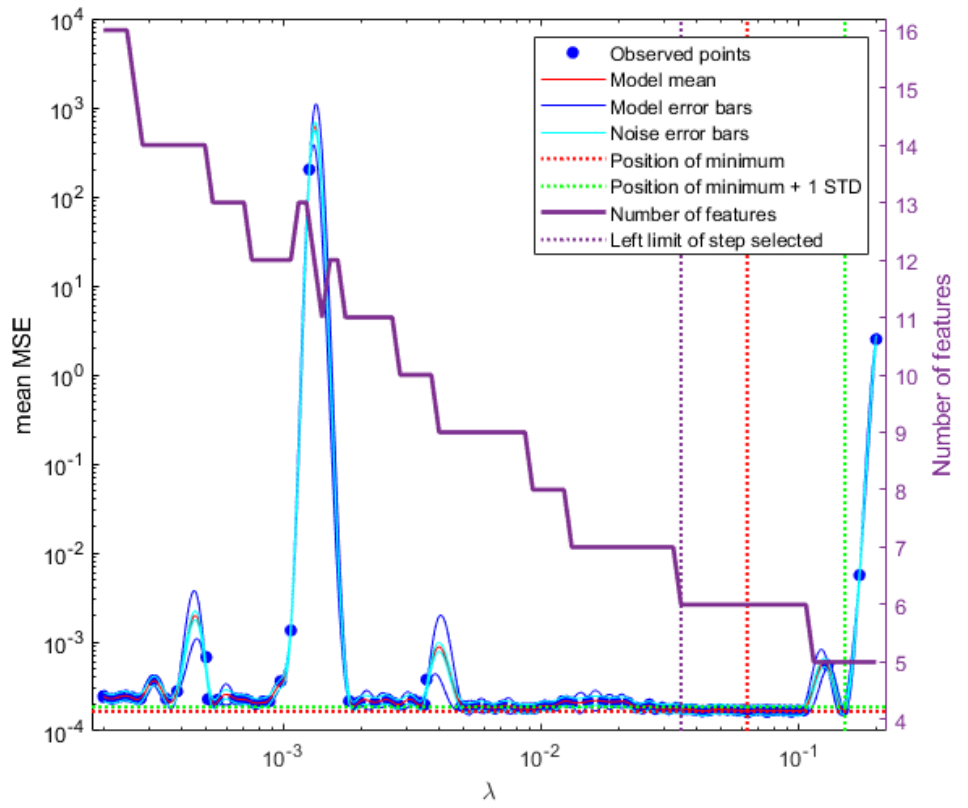

Figure 3. Selection of the value of $\lambda$ by BO based on $10000 \mathrm{CV}$ iterations at a fixed value of $\omega$.

The results are shown in Figure 3 for the parameter $\lambda$. The detailed explanation of the method will be given in an upcoming publication of ours, and the algorithm is planned to be made publicly available together with a user manual. For a given wavelength the results of the fit are presented in Figure 4.
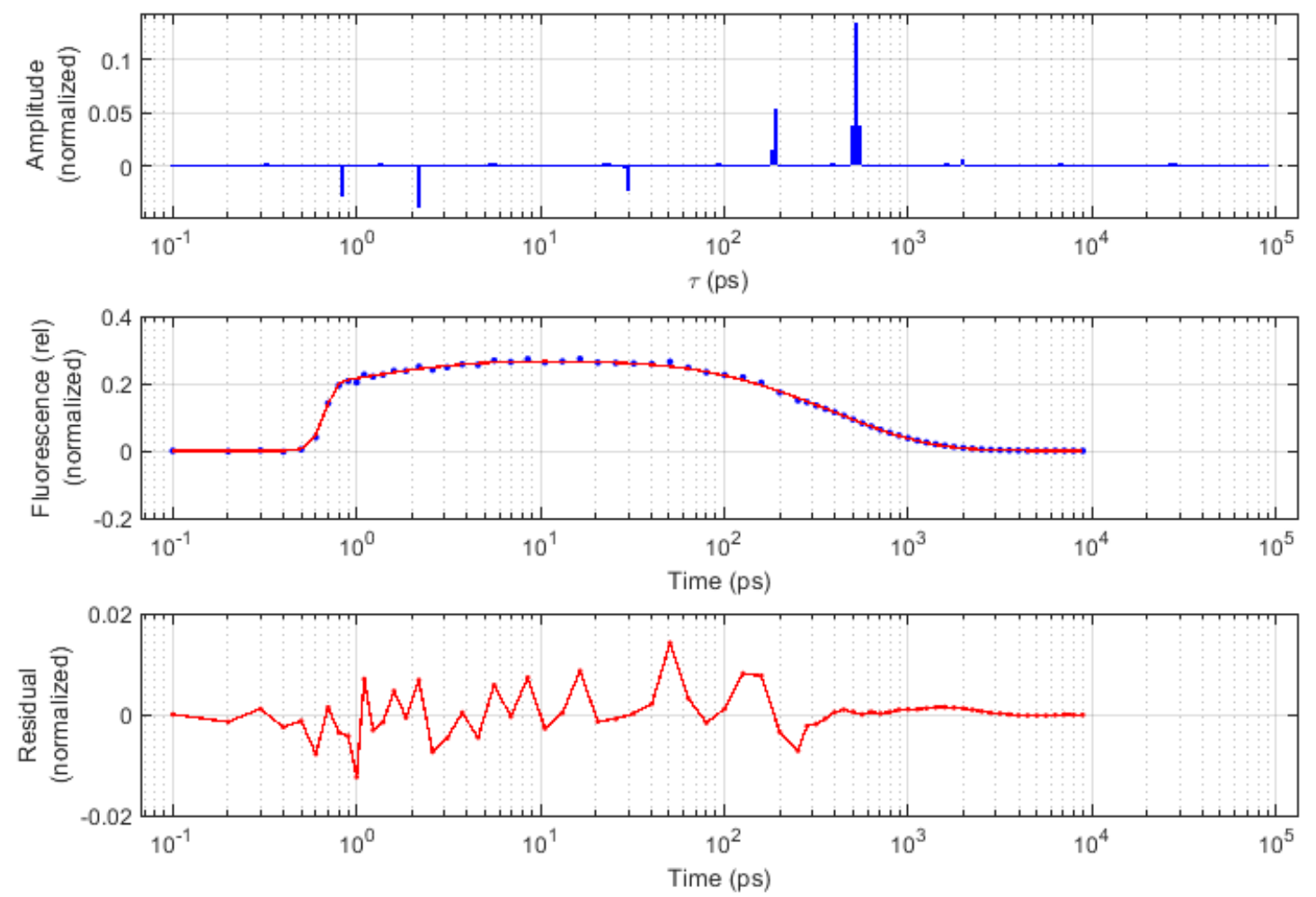

Figure 4. The distribution of time constants, the fit of data applying these time constants and the resulting error for observation at $510 \mathrm{~nm}$. 
As presented in Figure 5, the analysis resulted in several time constants observable in the fast (smaller than $100 \mathrm{ps}$ ) and in the slower (larger than $100 \mathrm{ps)} \mathrm{range.} \mathrm{The} \mathrm{slower} \mathrm{time} \mathrm{constants} \sim 200$ and $500 \mathrm{ps}$ are in accordance with the previous studies, the constant at $2000 \mathrm{ps}$ in negligibly small. The change from positive to negative values of the fast components with the increase of the wavelength can be explained by a time-resolved Stokes shift, related to a complex ultrafast vibrational relaxation phenomenon within the molecule.
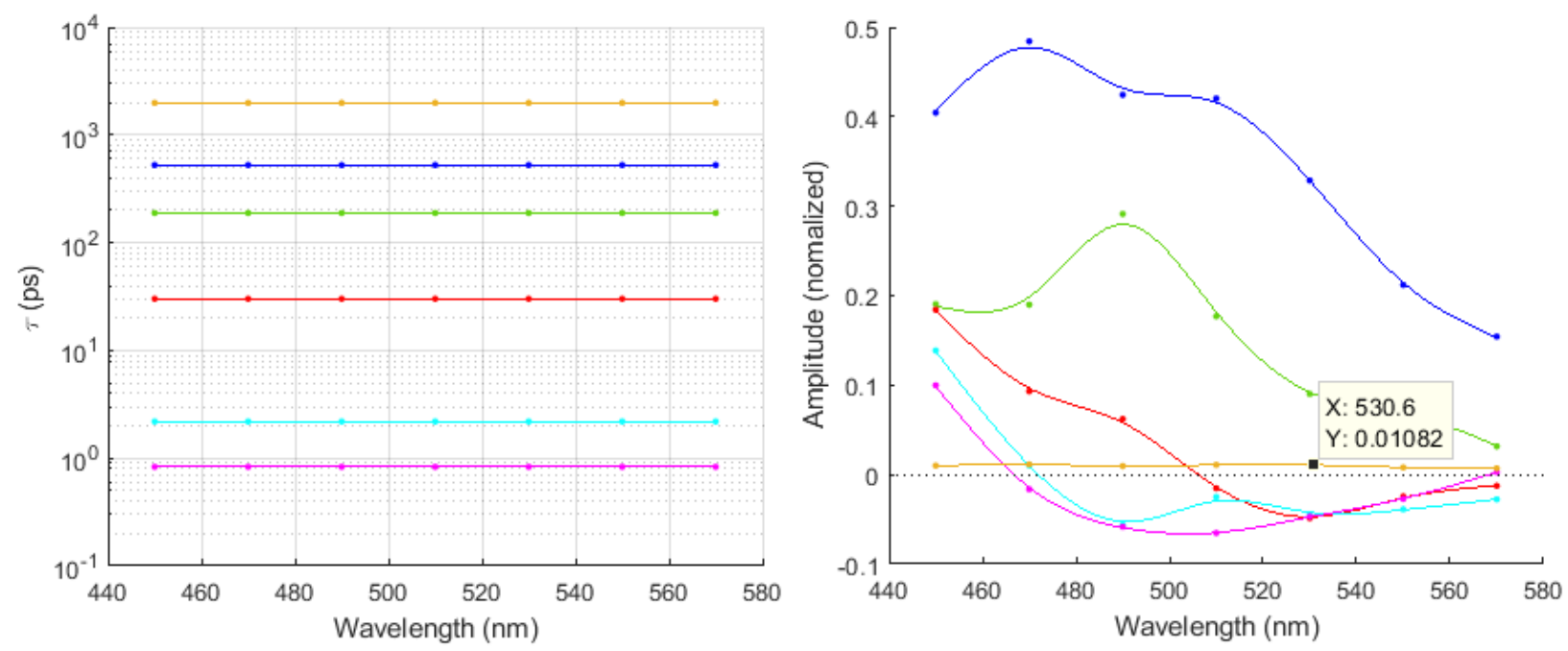

Figure 5. Time constrants (left) and their relative amplitude at different wavelengths (right).

\section{Conclusions}

We presented that if proper time resolution can be achieved in fluorescence kinetics measurement, and if modern statistical methods are applied to the analysis of the measured data, very fine details of the kinetics can be observed. In the case of NADH three ultrafast components obtained by such analysis can be associated with different vibrational relaxation processes of the molecule. The analysis method can be applied to any sufficiently detailed experimental data, opening a new way to study very fast dynamics in energy transfer related phenomena.

\section{Acknowledgements}

This work was supported by the National Research, Development and Innovation Office of Hungary under grants GINOP-2.3.2-15-2016-00001, 2018-1.2.1-NKP-2018-00009 and NKFIH PD-121170.

\section{References}

[1] Eto, K., Tsubamoto, Y., Terauchi, Y., Sugiyama, T., Kishimoto, T., Takahashi, N., Yamauchi, N., Kubota, N., Murayama, S., Aizawa, T., et al. (1999 (Washington, DC, U. S.) 283 (5404), 981-985.

http://doi.org/10.1126/science.283.5404.981

[2] Lehninger, A., Nelson, D. L., and Cox, M. M. (2008) Lehninger Principles of Biochemistry, W. H. Freeman.

[3] Koch-Nolte, F., Haag, F., Guse, A. H., Lund, F., and Ziegler, M. (2009). Sci. Signaling 2 , mr1. https://doi.org/10.1126/scisignal.257mr1

[4] Santidrian, A. F., Matsuno-yagi, A., Ritland, M., Seo, B. B., Leboeuf, S. E., Gay, L. J., Yagi, T., and Felding-habermann, B. (2013) J. Clin. Invest. 123 (3), 1068-1081.

https://doi.org/10.1172/JCI64264 
[5] Chance, B.; Cohen, P.; Jobsis, F.; Schoener, B. Science 1962, 137, 660. https://doi.org/10.1126/science.137.3531.660

[6] R. Hull, P. Conger III, R. Hoobler, Biophys. Chem. 90 (1) (2001) 9-16, https://doi.org/10.1016/S0301-4622(00)00239-8

[7] Meyer, W. L.; Mahler, H. R.; Baker, R. H. Biochim. Biophys. Acta, Gen. Subj. 1962, 64, 353-358.

[8] Cadena-Caicedo, A.; Gonzalez-Cano, B.; López-Arteaga, R.; Esturau-Escofet, N.; Peon, J. J. Phys. Chem. B 2020, 124, 519-530.

https://doi.org/10.1021/acs.jpcb.9b10012

[9] Freed, S.; Neyfakh, E. A.; Tumerman, L. A. Biochim. Biophys. Acta 1967, 143, 432-434.

[10] Heiner, Z.; Roland, T.; Leonard, J.; Haacke, S.; Groma, G. I. J. Phys. Chem. B 2017, 121, 8037-8045.

https://doi.org/10.1021/acs.jpcb.7b04753

[11] König, K.; Berns, M. W.; Tromberg, B. J. J. Photochem. Photobiol., B 1997, 37, 91-95. https://doi.org/10.1016/S1011-1344(96)07327-7

[12] Vishwasrao, H. D.; Heikal, A. A.; Kasischke, K. A.; Webb, W. W. J. Biol. Chem. 2005, 280, $25119-25126$.

https://doi.org/10.1074/jbc.M502475200

[13] Visser, A. J. W. G.; Hoek, A. v.. Photochem. Photobiol. 1981, 33, 35-40.

https://doi.org/10.1111/j.1751-1097.1981.tb04293.x

[14] Couprie, M. E.; Mérola, F.; Tauc, P.; Garzella, D.; Delboulbé, A.; Hara, T.; Billardon, M. Rev. Sci. Instrum. 1994, 65, 1485-1495.

https://doi.org/10.1063/1.1144880

[15] Ladokhin, A. S.; Brand, L. J. Fluoresc. 1995, 5, 99-106.

https://doi.org/10.1007/BF00718787

[16] Blacker, T. S.; Marsh, R. J.; Duchen, M. R.; Bain, A. J. Chem. Phys. 2013, 422, 184-194. https://doi.org/10.1016/j.chemphys.2013.02.019

[17] Pal, S. K.; Peon, J.; Zewail, A. H., Proc. Natl. Acad. Sci. U. S. A. 2002, 99, 1763-1768. https://doi.org/10.1073/pnas.042697899

[18] Ruhman, S.; Hou, B.; Friedman, N.; Ottolenghi, M.; Sheves, M. J. Am. Chem. Soc. 2002, 124, 8854-8858.

https://doi.org/10.1021/ja026426q

[19] Groma, G. I.; Heiner, Z.; Makai, A.; Sarlos, F., RSC Advances 2012, 2, 11481-11490.

https://doi.org/10.1039/C2RA21773B

[20] Chen, S. S. B.; Donoho, D. L.; Saunders, M. A., SIAM Journal on Scientific Computing 1998, 20, 33-61.

https://doi.org/10.1137/S1064827596304010 